\title{
First trimester fetal diagnosis of genetic disorders: clinical evaluation of 250 cases
}

\author{
BRUNO BRAMBATI*, GIUSEPPE SIMONI†, CESARE DANESINO \\ ANGELO OLDRINI*, ENRICO FERRAZZI*, LORENZA ROMITTI†, \\ GIANLUIGI TERZOLI†, FRANCA ROSSELLA†, MAURIZIO FERRARI§, \\ AND MARCO FRACCARO
}

From *Unità di Medicina Preventiva Perinatale and †Laboratorio di Citogenetica, I Clinica Ostetrica e Ginecologica, Università di Milano; $¥$ Istituto di Biologia Generale e Genetica Medica, Università di Pavia; and §Laboratorio di Ematologia, Istituti Clinici di Perfezionamento, Milano, Italy.

SUMMARY Chromosome and enzyme determinations were performed in 250 pregnancies between the 7th and the 12th week of gestation. The majority of the tests were performed for risk of chromosomal abnormalities and $75 \%$ of the women were 35 years old or more. We describe a chorionic villi sampling (CVS) technique which proved to be highly efficient, with a diagnostic success rate of $97.7 \%$. In the light of our experience we suggest that CVS is best performed between the 9 th and 10th weeks of pregnancy. The average weight of the aspirated specimen was $20 \mathrm{mg}$ with a lower limit of $5 \mathrm{mg}$ which proved sufficient for diagnostic purposes. No major maternal complications were encountered and the slight bleeding observed in $14 \%$ of the cases during the days following the CVS should be considered a harmless effect of the aspiration technique. The proportion of fetal losses may lie between 4 and $7 \%$. Paediatric monitoring of the 93 infants born so far and ultrasound examination of the pregnancies still in progress at the time of writing did not reveal any negative effect of CVS. Fetal-maternal transfusion and intrauterine infection are problems which need further basic investigations.

The methods for fetal diagnosis of genetic disorders in the second trimester of pregnancy are reliable and relatively safe and are an integral part of antenatal care. However, the obstetrical complications and the psychological constraint of selective abortion at around the 20th week of gestation are disadvantages that could be reduced by performing diagnosis in the first trimester. ${ }^{1-3}$. The Scandinavian trials ${ }^{4} 5$ first demonstrated the possibility of chorionic villi sampling (CVS), but an anonymous report from China was, until recently, the only clinical experience of first trimester fetal diagnosis. ${ }^{6}$ In an experimental study comparing four different sampling methods on a series of women scheduled for voluntary abortion we demonstrated that ultrasound guided villi aspiration is safe and efficient. ${ }^{7}$ It was therefore possible to introduce this sampling technique as a new antenatal diagnostic approach for fetal karyotyping and enzymatic tests.

In this paper we report the clinical evaluation of

Received for publication 29 July 1984. Accepted for publication 2 August 1984.
250 pregnancies in which the aspiration of chorionic villi, under direct ultrasound guidance, was used in the first trimester.

\section{Patients}

Among the 250 pregnancies, 228 were at risk for chromosomal abnormalities, 18 for fetal sex determination, and four for inborn errors of metabolism (table 1). The cases investigated for fetal karyotyping as the primary indication for CVS were 168 with maternal age of 35 years and over, 24 with maternal age under 35 years, 22 with a previous child showing a chromosome abnormality, and 12 with a chromosome abnormality in one parent. Fetal sex determination was performed in 13 cases at risk of $\mathrm{X}$ linked diseases. A further three cases had other genetic risks (campomelic dysplasia, congenital hip dislocation, and adrenogenital syndrome), and in two cases the primary indication followed psychiatric evalution. The four cases at risk for biochemical disorders included $\mathrm{GM}_{1}$ and gangliosidosis, MPS I 
TABLE 1 Results of fetal diagnosis in the first trimester by risk groups.

\begin{tabular}{|c|c|c|c|c|c|}
\hline Indication & $\begin{array}{l}\text { No of cases } \\
\text { investigated }\end{array}$ & $M / F$ & Abnormals & $\begin{array}{l}\text { Selective } \\
\text { abortions }\end{array}$ & Abnormalities found \\
\hline \multicolumn{6}{|l|}{ Chromosome abnormalities } \\
\hline Maternal age $\geqslant 35$ y & 168 & & 10 & 9 & $\begin{array}{l}3 \text { trisomy } 21.2 \text { trisomy } 18,2 \text { trisomy } 16,1 \text { trisomy } 14 \text {, } \\
1 \text { monosomy } X \| .1 \text { inv (X) in a male fetus }\end{array}$ \\
\hline Maternal age $\leqslant 35$ y & 24 & $108 / 118$ & - & - & \\
\hline $\begin{array}{l}\text { Previous child with } \\
\text { chromosome abnormality }\end{array}$ & 22 & & 3 & 3 & 1 trisomy 21.1 trisomy 13.1 mosaic with trisomy 3 \\
\hline $\begin{array}{l}\text { Chromosome abnormality in } \\
\text { the parents }\end{array}$ & 12 & & 6 & 2 & $\begin{array}{l}1 \text { trisomy } 22.1 \text { small metacentric marker and de novo } \\
\text { translocation. } 1 \text { balanced insertion, } 3 \text { Robertsonian } \\
\text { translocations }\end{array}$ \\
\hline \multicolumn{6}{|l|}{ Fetal sex determination } \\
\hline Risk of $\mathrm{X}$ linked diseases & $13^{*}$ & $4 / 8$ & 2 & $4 \S$ & 1 trisomy 21 (female), 1 trisomy 9 (female) \\
\hline Other genetic risk & 3 & $3 /-$ & - & - & \\
\hline Other reasons & 2 & -12 & - & 1 & \\
\hline Enzyme determinations & 4 & $2 / 2$ & $1 \ddagger$ & 1 & 1 MPS I \\
\hline Totals & $248+$ & $117 / 130$ & 22 & 20 & \\
\hline
\end{tabular}

*One diagnostic failure.

†Villus material was not available for investigation in five cases. The total is inclusive of three twin pregnancies. Ten diagnoses were performed at III Clinic of Obstetrics and Gynecology of Milan University by Drs L Dalprà. M G Tibiletti, and G Nocera.

\$MPS I diagnosed at 19 weeks on cultured amniocytes.

\$One pregnancy from a woman with a brother with Duchenne muscular dystrophy is continuing in spite of diagnosis of a male fetus. In a pregnancy with a male

fetus a second trimester diagnosis of haemophilia A was performed at King's College Hospital, London.

$\|$ At ultrasound performed one week after sampling the intrauterine death of the fetus was revealed.

This summary of the results includes the 100 cases reported by Simoni et al. ${ }^{9}$

Hurler's syndrome, Niemann-Pick's disease, and metachromatic leucodystrophy.

The gestational age at the time of sampling was between the 7 th and the 12th week. The maternal age groups were 20 to 24,25 to 29,30 to 34,35 to 39 , 40 to 44 , and 45 or more in $3 \%, 6 \%, 16 \%, 38 \%$, $35 \%$, and $2 \%$ of cases, respectively.

Each patient was requested to sign an informed consent form presenting amniocentesis and CVS as alternative choices and specifying that the obstetric risk of first trimester fetal diagnosis was not as yet defined.

\section{Methods}

Sampling of chorionic tissue was done with a catheter $1.5 \mathrm{~mm}$ in diameter and $15.5 \mathrm{~cm}$ long (Portex Ltd, England). A mechanical sector scanner (ATL MK 300) with a $5 \mathrm{MHz}$ transducer was used to visualise the uterus and to guide the aspiration catheter as previously described. ${ }^{7}$ A transabdominal, ultrasonically guided biopsy technique was also used in selected cases introducing a 20 gauge needle in the plane of the ultrasound section near the transducer.

During the second and third trimester the fetus was monitored by a real time linear array scanner with a $3.5 \mathrm{MHz}$ transducer. No premedication or local anaesthesia were necessary because the sampling procedure proved painless. ${ }^{7}$ A strict medical protocol was established. At the first visit the genetic counselling included information about the procedure and the potential danger of CVS, emphasising the limited experience regarding the obstetric and fetal risks. A vaginal swab was taken in the first 100 cases and topical therapy given during the four days preceding CVS in the event of positive culture. The remaining 150 cases received vaginal treatment with povidone-iodine suppositories (Betadine) as a prophylactic measure. The culture techniques used were appropriate for isolating the following organisms: Gardnerella vaginalis, Neisseria gonorrhoeae, Streptococcus, Micrococcus, Enterobacter, Trichomonas vaginalis, Candida albicans. Finally, echographic examination was used to assess the viability of the pregnancy and to evaluate fetal age. At sampling, the external genitalia were cleaned with Betadine solution, and the cervix was visualised with a Grave speculum and grasped by a tenaculum. A swab was taken from the external cervical os.

A probe (hysterometer) with a rounded tip of about $3 \mathrm{~mm}$ diameter was introduced first to evaluate the cervical canal and to detect on the ultrasound display the internal cervical os and its relationship with the lowest margin of the placenta. From case 47 onwards, the identification of the umbilical cord insertion on the chorionic plate was used systematically to localise the placental site.

In the case of unsuccessful or insufficient sampling no more than four catheter insertions were performed. Blood samples were drawn for maternal serum AFP measurements just before and one to two hours after CVS. The serum AFP was measured 
by radioimmunoassay. A further evaluation of the maternal AFP level was conducted at 16 to 18 weeks. An ultrasound check was made one to three hours after CVS before the patient was allowed to leave the hospital.

In patients at risk for Rhesus isoimmunisation an indirect Coombs test was done before CVS and, if negative, $200 \mu \mathrm{g}$ anti-D immunoglobulin were given in the 72 hours after sampling.

Seven to ten days after CVS an ultrasound study was performed to verify the viability and the growth of the fetus and to follow up the evolution of any echo-free space previously detected.

During the fifth month of pregnancy ultrasound examination was undertaken especially to detect gross fetal abnormalities, and the rate of fetal growth was established in the seventh or eighth month.

Termination of pregnancy, in cases of selective abortion, was carried out under general anaesthesia by aspiration technique the day following the diagnosis of an abnormal fetus.

Fetal chromosomes were obtained by the direct method. ${ }^{9}$ The villi were washed in Hank's saline balanced solution and then transferred to RPM-I medium without serum. Colcemid was added to the medium at $0.04 \mu \mathrm{g} / \mathrm{ml}$ final concentration for one hour. A $1 \%$ sodium citrate solution was used for hypotonic treatment (10 minutes) and 3:1 methanolacetic acid for fixation (10 minutes). The villi were then dissociated with $60 \%$ acetic acid solution. Chromosome preparations were made by distributing the cell suspension onto the surface of warm slides $\left(40^{\circ} \mathrm{C}\right)$ using a bent Pasteur pipette. The slides were routinely stained for QFQ banding. disorders were selected and suspended in $0.5 \mathrm{ml}$ distilled water, minced with scissors, frozen and thawed five times, then centrifuged for 20 minutes at $4000 \mathrm{rpm}$.

The resulting supernatant was frozen at $-20^{\circ} \mathrm{C}$ until enzymatic assay. ${ }^{7}$ Methods of assay for lysosomal enzymes were those reported by Galjaard $^{10}$ with minor modifications.
Chorionic villi from patients at risk for metabolic

\section{Results}

Table 2 summarises the results from February 1983 to May 1984. CVS in the first trimester was offered to 250 women, including three twin pregnancies, and produced a total of 248 successful samplings. In six cases we failed to obtain chorionic tissue, and the procedure was stopped in four of them at the second attempt and in two at the fourth attempt. Amniocentesis in the second trimester was offered to all these cases. However, CVS was repeated in one patient seven days later at her specific request. This time CVS was performed by transabdominal aspiration. The overall success rate was $97.7 \%$ (table 3). The weight of the aspirated chorionic villi specimens ranged from 5 to $100 \mathrm{mg}$. CVS was carried out at the 7th, 8th, 9th, 10th, 11th, and 12th week in 2, 24, 96, 98, 25 and 6 cases, respectively. Selective abortion was undertaken in 20 cases. Two voluntary abortions for non-genetic reasons were requested weeks after an uneventful CVS and a normal fetal karyotype. In the first case, in which a threatened abortion followed by a chronic AF

TABLE 2 Summary of results of chorionic villi sampling at Mangiagalli Clinic, Milan, February 1983 to May 1984.

\begin{tabular}{lr}
\hline No of patients & 250 \\
Twin pregnancies & 3 \\
Repeated sampling & 1 \\
Total no of samplings & 254 \\
Failed sampling & 6 \\
Amniocentesis & 8 \\
Selective abortion & 20 \\
Non-genetic abortion & 2 \\
Total no of voluntary abortions & 23 \\
Pregnancies intended to continue & 227 \\
& \\
Fetal losses & \\
$\quad$ After 1 to 2 weeks & 5 \\
After 3 to 6 weeks & 2 \\
After lo weeks & 2 \\
Total & 9 \\
Delivered & 89 \\
$\quad$ After 37 weeks & 4 \\
Before 37 weeks & 93 \\
Total & \\
\hline
\end{tabular}

TABLE 3 Distribution of rates of attempt and failure. Starting from case 47 identification of the umbilical cord insertion was used to localise the placental site (see methods).

\begin{tabular}{|c|c|c|c|c|c|c|}
\hline Successful sampling & At the 1st attempt & At the 2nd attempt & At the $3 r d$ attempt & At the 4th attempt & Failed sampling & Total no of cases \\
\hline From case 1 to case 46 & $\begin{array}{c}28 \\
(62)\end{array}$ & $\begin{array}{l}10 \\
(22)\end{array}$ & 3 & 4 & $\begin{array}{l}1 \\
(2 \cdot 1)\end{array}$ & 46 \\
\hline Total & $\begin{array}{l}208 \\
(82)\end{array}$ & $\begin{array}{l}31 \\
(16)\end{array}$ & 4 & 5 & $\begin{array}{l}6 \\
(2 \cdot 3)\end{array}$ & $254^{*}$ \\
\hline
\end{tabular}

*Three twin pregnancies and one repeated CVS. 옥

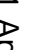
. 
leakage occurred, the couple decided for psychological reasons to abort at the 22 nd week. The amount of amniotic fluid was apparently not reduced and the fetus was developing normally. The second case, a twin pregnancy, was a 44 year old women with six living children. Two weeks after CVS at 12 to 13 weeks the couple decided to abort for social reasons.

Amniocentesis in the second trimester was performed in seven cases: in three because of CVS failure, and in the others to confirm ambiguous diagnostic results.

The first 100 pregnancies to be continued were monitored to term; 89 cases delivered after the 37 th week and four were delivered preterm by caesarean section at $30,32,35$, and 36 weeks, respectively. The indication for premature delivery was a preeclamptic state in three and acute fetal distress detected in a patient with hypertension and diabetes. The birth weights were between the 10th and 90 th centiles in 85 cases. $^{11}$ In two cases they were above the 90th centile, and in six cases below the 10 th centile. Among the mothers of the small babies pre-eclampsia was present in three cases and essential hypertension and diabetes in one case. The Apgar score calculated at five minutes in 75 cases was 8 to 10 . One baby developed mild jaundice and anaemia because of an $\mathrm{ABO}$ erythroblastosis fetalis. A mild form of talipes equinovarus occurred in one case. The placental morphology of the 89 uncomplicated pregnancies delivered at term was reported to be normal and the placental weight was consistent with the size of the newborn.

At the time of writing 125 pregnancies are continuing uneventfully and the fetal growth observed by ultrasound measurements is within our normal limits.

Light bleeding after CVS was reported by the patient in 35 cases (14\%) during the first 24 to 72 hours. In three other cases the bleeding persisted for two to three weeks. An echo-free space was detected in the extraplacental area in two of these three patients two hours after sampling, which disappeared some weeks after the bleeding stopped. In five cases slight bleeding appeared nine to 30 days after CVS and persisted for one to two days. Chronic amniotic fluid leakage followed a threatened abortion in three cases at 10, 16, and 17 weeks (cases 65, 16, and 102). Cases 16 and 65 obtained a voluntary abortion and case 102 aborted spontaneously.

Fetal loss was observed in nine cases and the clinical aspects are described in table 4 . Seven cases occurred among the first 100 cases followed to term. In two of the five intrauterine deaths (cases 54 and 144) a chromosomal aberration was present in the fetus. In case 54 the sampling was unsuccessful and trisomy 14 was diagnosed by karyotyping the fetal tissue after evacuation. In case 65 , which had a bad prognosis because of marked oligohydramnios, the fetus was alive and growing normally at the time of voluntary abortion. Ultrasound examinations of cases $4,8,21,75,102$, and 195 performed just before the fetal loss were normal. No abnormalities were detected in cases 8,21 , and 65 , in which the fetal material was suitable for morphological study. In three cases small clots of 0.25 to $0.50 \mathrm{cc}$ were noted at the edge of the placental plate after uterine evacuation.

Vaginal swabs for bacteriology four days before CVS and immediately before sampling were negative in 29 out of 100 cases. In the remaining 221 cases in which intravaginal treatment preceded CVS, $75(34 \%)$ had positive cervical cultures, Streptococcus, Enterobacter, Candida albicans, and Gardnerella vaginalis being the most frequently found. In two of the nine fetal losses (cases 8 and 54)

TABLE 4 Details of the nine cases of fetal loss.

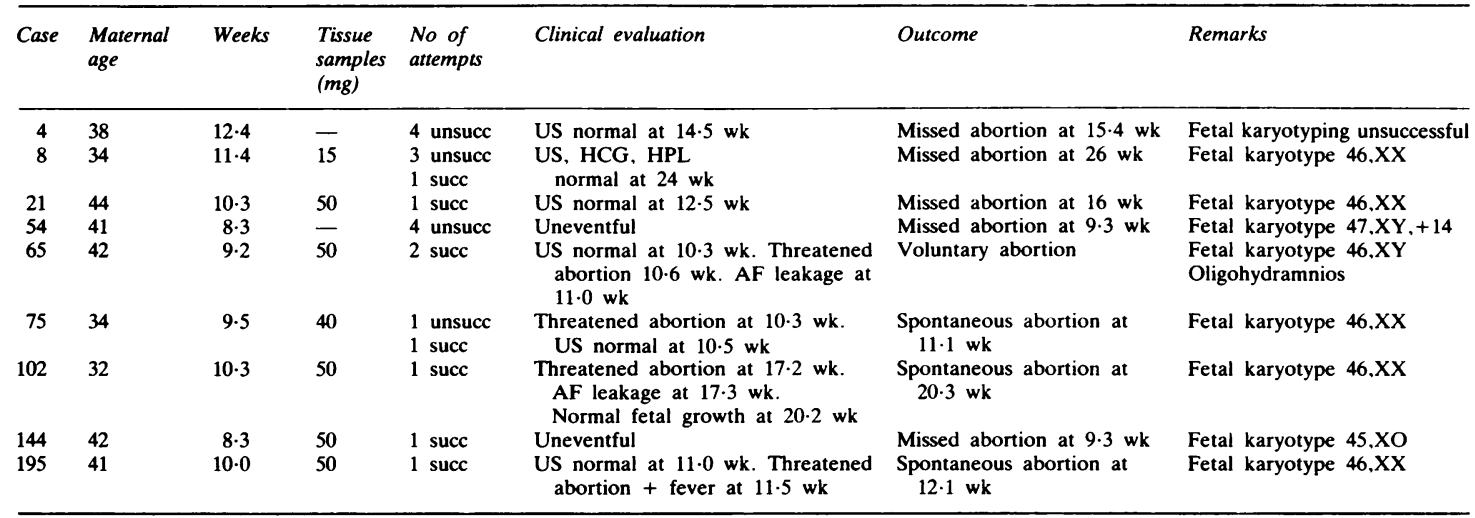


the cervical culture before CVS was positive for Proteus mirabilis and Enterococcus, respectively. No bacterial growth was present in the seven other cases, including case 195 who was suspected clinically of having an intrauterine infection. Similarly, negative results were obtained in four cases of fetal loss (cases 8, 54, 65, and 75) culturing fetus or membranes or both after evacuation.

Five $R h$ negative patients delivered $R h$ positive babies. All of them received anti-D serum following CVS and none developed antibodies. Eight group O women had seven group A infants and one group B. In three cases anti-A antibodies appeared for the first time in the third trimester. In one case the newborn required phototherapy. No modifications of fetal heart rate were observed by real-time ultrasound performed immediately after CVS.

The average time interval between CVS and laboratory diagnosis was three days, ranging from three hours to seven days. Fetal chromosome studies were successful in 247 out of 248 cases in which villi were obtained at sampling. The diagnostic failure occurred where the sample was good but the metaphases were of poor quality. As shown in table 1 , an abnormal chromosome constitution was found in 21 cases. Of these, 16 were aneuploid, three were Robertsonian translocations, one was a balanced insertion inherited from the father, and one was an $\operatorname{inv}(X)$ in a male fetus. Two trisomies were found in female fetuses from pregnancies at risk for $\mathrm{X}$ linked disorders. The cytogenetic analysis confirmed the diagnosis in 14 out of 16 cases with abnormal karyotypes in which a selective abortion was performed. The abnormal chromosome constitution was also confirmed in the case with monosomy $\mathrm{X}$, in which intrauterine death of the fetus occurred one week after sampling.

In two cases the abnormal fetal karyotype detected by the direct method was not confirmed after termination of the pregnancy. These were a mosaic with trisomy 3 and a trisomy 16 . Both abnormalities were confined to the chorionic tissue while a normal chromosome constitution was consistently found in fetal fibroblast cultures obtained from at least two biopsies in each case.

First trimester fetal diagnosis was offered to five couples at risk for metabolic diseases. However, prospective fetal diagnosis was performed in only four of them, because a missed abortion occurred at eight weeks in the case at risk for Tay-Sachs disease. The direct enzymatic analysis of chorionic tissue obtained by aspiration just before the uterine evacuation excluded Tay-Sachs disease by the presence of hexosaminidase A and B. ${ }^{12}$ In two families at risk for Niemann-Pick disease and gangliosidosis $\mathrm{GM}_{1}$, normal levels of activity indicated two un- affected fetuses. The two children have been born, and at six months of age they are clinically healthy. Confirmation of the diagnosis on leucocytes is in progress. In the case at risk for Hurler's syndrome $\overrightarrow{0}$ the result obtained from chorionic villi, indicating a $\overline{0}$ healthy fetus, was not confirmed when amniotic $\frac{\bar{\sigma}}{\bar{\rho}}$ fluid cells were assayed. Fibroblasts from the $\vec{\nabla}$ aborted fetus in fact confirmed the absence of $\alpha$-iduronidase. ${ }^{9}$

Normal levels of activity of arylsulphatase A have $\overrightarrow{0}$ been found in chorionic villi of the case at risk for $\overrightarrow{-}$ metachromatic leucodystrophy; the assay will be $\vec{\omega}$ repeated on cultured amniocytes to confirm the diagnosis.

\section{Discussion}

EFFICIENCY OF THE SAMPLING TECHNIQUE The overall success rate of more than $96 \%$ confirmed the expectation derived from the ex- $D$ perimental study. ${ }^{7}$ The few sampling failures were due to some specific conditions for which the sampling technique should be considered inadequate. These were mainly the following: (a) the placental tissue is hardly outlined before the eighth week; (b) the implantation site was too far (more than $16 \mathrm{~cm}$ ) from the vaginal entrance, mainly observed from the 11th week onwards; and (c) the angle between the cervical axis and the intrauterine path to the placenta was unusually pronounced.

A longer catheter could allow a successful sampling in condition (b), as could the use of a rigid device or transabdominal aspiration in condition (c). In any case, to reduce the inconvenience of unsuccessful introduction of the catheter it seems appropriate to limit the sampling to the ninth and tenth weeks and to perform CVS later in the pregnancy only after an accurate evaluation by the combined use of ultrasound and the hysterometer. In the present experience $78 \%$ of samples were taken from nine to ten weeks, while the number of cases sampled between the seventh and eighth week was small $(10 \%)$ and confined to the beginning of the study. Recently, a limited number of selected or cases $(12 \%)$ was delayed and sampled at 11 and $12 N$ weeks because of growing demand and limited $\underset{\omega}{\mathrm{N}}$ diagnostic resources.

With increasing experience the umbilical cord insertion to the placenta was identified in more than $90 \%$ of the cases and introduced as a specific $\stackrel{\oplus}{\rightarrow}$ indicator of the sampling site. Although in the last 0 104 cases the overall success rate by these new sampling criteria remained nearly unchanged (table 3 ), the number of cases in which more than one aspiration attempt was necessary to obtain chorionic villi was reduced from $38 \%$ to $14 \%$. 
The aspiration procedure gave consistent amounts of chorionic tissue sufficient for all diagnostic purposes. In only three cases a second specimen was requested during the same procedure to set up long term culture of chorionic villi cells.

FETAL LOSS AND OBSTETRIC COMPLICATIONS A crude fetal loss rate of $3.9 \%$ could be estimated including the 125 cases still in progress at the time of writing, 72 of which are over 20 weeks' gestation. However, the follow up to term of the first 100 cases provided a correct rate of $7 \%$. No case of fetal loss was observed within seven days of sampling. A direct relationship between the sampling procedure and the abortion was postulated in cases 75 and 195 . In case 75 , bleeding and uterine contractions started on the third day and a complete abortion took place on the tenth day. The patient complained of suprapubic pains during the two weeks preceding CVS. The fever present in case 195 at the same time as vaginal bleeding and uterine contractions was suspected to be the consequence of bacterial invasion of the uterine cavity following CVS, although the cervical culture at sampling was negative and only one uncomplicated aspiration was made. In cases 54 and 144 the fetal death detected at the scheduled ultrasound check one week after CVS strongly suggested a relationship with the chromosomal abnormalities found in the fetus. In the other seven cases there was no evidence of injury at the time of CVS, such as haemorrhage or membrane perforation, or post mortem evidence of damage to the placenta. Although no obvious connection could be established between the fetal loss and the number of times the catheter was introduced, in three of the first four fetal losses the catheter insertion was repeated four times. Thus, we decided to limit the aspiration attempts to a maximum of two to minimise placental trauma and the likelihood of bacterial invasion. The gestational age at sampling and the total amount of chorionic villi aspirated were apparently not related to the abortion rate. It should be noted that all six chromosomally normal fetuses lost after CVS were female.

No direct perforation of the ovular sac was detected soon after sampling. The amniotic fluid leakage in cases 16, 65, and 102 should be interpreted as a late sign of threatened abortion rather than as a direct consequence of the procedure. In all three cases villi were sampled at the first attempt, and the procedure reviewed on videotape did not show threatening movements of the tip of the catheter. Nevertheless, some direct undetectable trauma reducing the resistance of the chorion and favouring a spontaneous rupture cannot be ex- cluded, nor can the concurrence of an intrauterine infection.

The slight or moderate bleeding which occurred in several cases during the first two days had no adverse effect and should be considered as a harmless consequence of placental perforation during the aspiration of chorionic tissue.

Considering the clinical and ultrasound evidence for the 93 cases which came to term and for the remaining cases still in progress, no adverse findings were observed in the mother or the fetus which could be attributed to the CVS procedure.

Two further aspects of first trimester fetal diagnosis should be evaluated during the current applied research phase, namely the fetomaternal haemorrhage and the intrauterine infections as a consequence of the aspiration procedure.

\section{FETOMATERNAL HAEMORRHAGE}

Fetomaternal transfusion was postulated as a possible consequence of the CVS and was checked by the variation of AFP levels in maternal serum samples taken before and after sampling. The preliminary analysis of the data seems to indicate a significant change in the maternal serum AFP after CVS in a large proportion of cases. In over $50 \%$ where more than $50 \mathrm{mg}$ of chorionic villi were aspirated a significant rise in maternal AFP level was demonstrated. In the Rhesus negative mothers at risk of $\mathrm{Rh}$ isoimmunisation and without antibodies, anti-D immunoglobulins were administered to avoid immunisation. To date, the follow up of the fetuses and infants of the 16 patients treated with $200 \mu \mathrm{g}$ anti-D immunoglobulin after CVS is in progress and no developmental problems have been seen which could be related to anti-D serum prophylaxis. However, the anti- $\mathrm{A}$ antibodies which appeared late in the pregnancy in the three women at risk for ABO incompatibility could be a consequence of fetomaternal transfusion following chorionic villi aspiration, although in these cases no significant differences in maternal AFP levels before and after sampling were detectable.

\section{INTRAUTERINE INFECTIONS}

Although our data suggest that there is only a very limited risk of acute intrauterine infection following CVS, bacterial invasion of the uterine cavity may play an aetiological role in the later complications of pregnancy, such as preterm rupture of membranes or fetal death in utero. ${ }^{13-15}$ No clinical signs were observed in the nine patients with fetal loss, and the bacteriological study undertaken in four of them on the fetus or membranes or both was inconclusive. The high rate $(36 \%)$ of positive cultures of cervical swabs just before CVS and after antimicro- 
bial prophylaxis highlights the low efficacy of the vaginal treatment. In this connection, we lack comprehensive knowledge of the ecology and significance of the vaginal flora and of the effect of antibiotics on it. At the same time we need knowledge on the teratogenic or toxic effect on the fetus of a series of antimicrobial substances that are or could be used for vaginal treatment.

\section{CONTRAINDICATIONS}

The presence of $\mathrm{Rh}$ immunisation should be considered as an absolute contraindication to CVS: the fetal blood frequently detected in the maternal stream could be sufficient to stimulate maternal immunoresponse, worsening the prognosis of the pregnancy in cases of fetomaternal incompatibility.

A twin pregnancy with fused placentas should be a contraindication to the use of the aspiration technique whenever the umbilical cord insertions are not identified, since in this case samples from the two different chorionic tissues may not be selected with confidence. ${ }^{16}$ Acute vaginal infections, bleeding, or uterine cramps just before sampling are indications for deferring the procedure.

\section{DIAGNOSTIC PROBLEMS}

Because of the early stage of pregnancy at CVS one is bound to find those abnormal karyotypes which are usually found among spontaneous abortions, but not in the second trimester. The major diagnostic problem encountered was the occurrence of a trisomy 3 mosaicism and a trisomy 16 revealed by the direct procedure and strictly confined to villus tissues, confirming the possibility of a discrepancy between cytotrophoblast cells and fetal fibroblasts. ${ }^{17}$

On these grounds we think that additional investigations in the second trimester should be offered either when a mosaic is found in the villus or when a rare type of autosomal trisomy, compatible only with minimal embryonic development, is found in a pregnancy with apparently normal growth of the conceptus. In these cases, if the abnormal fetal karyotype is not confirmed in the second trimester and the fetus is developing normally as revealed by ultrasound monitoring, a normal chromosome constitution may be predicted with a high degree of confidence.

Finally, the occurrence of two trisomic female karyotypes out of 13 pregnancies at risk of $X$ linked disorders demonstrates that even if procedures for first trimester fetal sexing were available using specific DNA sequences, ${ }^{18} 19$ chromosome analysis cannot be omitted.

The wrong diagnosis made in the case at risk for Hurler's disease, which was corrected at amniocentesis, was most likely due to a technical error.
However, at least in theory, the possibility cannot be excluded of the existence of real differences between fetal and chorionic cells due to the presence in the latter of active isoenzymes not detectable in the former.

Two other cases are known in which the results obtained in chorionic villi homogenates were not confirmed when amniocytes or even cultured cells from villi were assayed ${ }^{20}$ (R J A Penketh, 1984, personal communication).

\section{Conclusions}

We feel that our experience, although limited, offers the following conclusions.

(1) The sampling technique was consistently reliable.

(2) No immediate major complications were found.

(3) No adverse affects on growth and development of the fetus were observed.

(4) The proportion of fetal losses, which is estimated at the time of writing as 4 to $7 \%$, is in the range of the spontaneous abortion rate expected for pregnancies between the second and third month of gestation. ${ }^{21}$

The risk of diagnostic errors appears to be very low. Nevertheless, the occurrence of two discrepancies between placental cells and fetal fibroblasts emphasises the need for more data in order to evaluate the incidence of this phenomenon and its clinical significance.

Our results indicate that CVS for the diagnosis of genetic diseases in the first trimester is a safe and reliable procedure with an apparently low risk for the fetus. However, significant risk figures must wait until international collection of extensive data is achieved.

\section{References}

1 Cates W Jr, Grimes DA. Deaths from second trimester abortion by dilatation and evacuation: causes, prevention, facilities. Obstet Gynecol 1918;58:401-8.

${ }^{2}$ Donnai P, Charles N, Harris R. Attitudes of patients after 'genetic' termination of pregnancy. Br Med J 1981;282:621-2.

${ }^{3}$ Greer HS, Lal S, Lewes SC, Belsey EM, Beard RW. Psychological consequences of therapeutic abortion. Kings termination study III. Br J Psychiatry 1976;128:74-9.

${ }^{4}$ Kullander S, Sandahal B. Fetal chromosome analysis after transcervical placental biopsies during early pregnancy. Acto Obstet Gynaecol Scand 1973;52:355-9.

5 Hahnemann N. Early prenatal diagnosis; a study of biopsy techniques and cell culturing from extraembryonic membranes. Clin Genet 1974;6:296-306.

6 Anshan Department of Obstetrics and Gynecology. Fetal sex prediction by sex chromatin of chorionic villi cells during early pregnancy. Chin Med J 1975;1:117-26.

7 Simoni G, Brambati B, Danesino C, et al. Efficient direct chromosome analysis and enzyme determination from chorionic villi samples in the first trimester of pregnancy. Hum Genet 1983;63:349-57. 
${ }^{8}$ Brambati B, Simoni G. Diagnosis of fetal trisomy 21 in first trimester. Lancet 1983:i:586.

9 Simoni G, Brambati B, Danesino C, et al. Diagnostic application of first trimester trophoblast sampling in 100 pregnancies. Hum Genet 1984;66:252-9.

${ }^{10}$ Galjaard H. Genetic metabolic diseases. Amsterdam: Elsevier/ North Holland, 1980.

11 Bossi A, Caccamo ML, Scrilli AM, Milani S. Weight of the Italian newborn infants (from the 32nd to 43rd week of gestation). Riv Ital Pediatr 1980;6:153-70.

12 Danesino C. Enzyme determinations on chorionic villi in the first trimester of pregnancy for prenatal diagnosis of metabolic diseases. In: Albertini A, Crosignani PG, eds. Progress in perinatal medicine. Amsterdam: Excerpta Medica, 1983.

${ }^{13}$ Naeye RL. Causes of perinatal mortality in the US Collaborative Perinatal Project. JAMA 1977;237:228-9.

14 Bejar R, Curbello V, Davis C, Gluck L. Premature labor. II. Bacterial sources of phospholipase. Obstet Gynecol 1981;57:479-82.

15 Minkoff H. Prematurity: infection as an etiologic factor. Obstet Gynecol 1983;62:137-44.
${ }^{16}$ Brambati B, Oldrini A, Simoni G, et al. First trimester fetal karyotyping in twin pregnancy. $J$ Med Genet 1984;21:58-60.

${ }^{17}$ Kalousek DK, Dill FJ. Chromosomal mosaicism confined to the placenta in human conceptions. Science 1983;221:665-7.

18 Gosden JR, Mitchell AR, Gosden CM, et al. Direct vision chorionic biopsy and chromosome specific DNA probes for determination of fetal sex in first trimester prenatal dignosis. Lancet 1982;ii:1416-9.

19 Williams H, Bown CS, Thomas NST, et al. First trimester fetal sexing in pregnancy at risk for Duchenne muscular dystrophy. Lancet 1983;ii:586-9.

${ }^{20}$ Fensom AH, Jackson M, Sanguinetti N, et al. The use of chorionic villi for early prenatal diagnosis of metabolic disorders. J Med Genet 1984;21:142A.

${ }^{21}$ Gustavii B. Chorionic biopsy and miscarriage in first trimester. Lancet 1984;: 562 .

Correspondence and requests for reprints to Dr B Brambati, Unità di Medicina Preventiva Perinatale, I Clinica Ostetrica e Ginecologica, Università di Milano, Milano, Italy. 